\title{
Utilização de estrógeno exógeno no início do ciclo estral em vacas leiteiras mestiças
}

\author{
José Rogério Moura Almeida Neto ${ }^{1}$, Eduardo Paulino da Costa ${ }^{2}$, Ademir de Moraes \\ Ferreira $^{1,3}$, Wanderlei Ferreira de Sá $^{3}$, Giancarlo Magalhães dos Santos ${ }^{2}$, Rafael José Otero \\ Arroyo $^{2}$
}

${ }^{1}$ Faculdade de Medicina Veterinária de Valença (FMVV/CESVA) - Valença-RJ.

2 Departamento de Veterinária - UFV - Viçosa, MG.

${ }^{3}$ Embrapa Gado de Leite - Juiz de Fora, MG.

RESUMO - O objetivo neste trabalho foi verificar o efeito do estrógeno exógeno em fase precoce do ciclo estral na indução do estro e na dinâmica ovariana de vacas leiteiras. Considerando que a PGF2 $\alpha$ não regride o corpo lúteo com menos de cinco dias após o estro, uma das alternativas para a redução do intervalo estral no início do ciclo seria o uso do estrógeno. Foram utilizadas 16 vacas mestiças Holandês-Zebu ciclando regularmente, sem qualquer alteração clínica ou reprodutiva. As vacas foram incluídas ao acaso nos respectivos tratamentos. Oito delas receberam $2,5 \mathrm{~mL}$ de cipionato de estradiol no primeiro dia do ciclo estral e oito não receberam (controle). A manifestação de estro foi monitorada visualmente. Os exames ultrassonográficos foram realizados diariamente pela manhã, iniciando no dia do estro. As coletas de sangue para as dosagens de progesterona tiveram início no dia do estro. Os animais que receberam estrógeno no primeiro dia após o estro natural manifestaram os sinais característicos de estro (psíquicos, útero túrgido e muco abundante) um dia após a aplicação. Essa redução do intervalo de estro para apenas dois dias pode favorecer a eliminação de bactérias do útero (quando presentes), aumentando a eficiência reprodutiva. Excetuando o dia da emergência da primeira onda folicular, todas as outras variáveis estudadas (número e comprimento de ondas, características dos folículos dominantes e subordinados, assim como os parâmetros relacionados ao corpo lúteo) não foram afetadas pela aplicação de estrógeno um dia após o estro. A aplicação de cipionato de estradiol em vacas mestiças um dia após o estro natural promove o aparecimento de sinais de estro dois dias após o estro natural mas não afeta as características do ciclo estral subsequente.

Palavras-chave: hormônio, reprodução, vacas

\section{Use of exogenous estrogen in the beginning of estrous cycle in crossbred dairy cows}

\begin{abstract}
The objective of this work was to check the effect of exogenous estrogen, in early phase of estrus cycle on the induction of estrus and on ovarian dynamics of dairy cows. By considering that PGF2 $\alpha$ does not regress the corpus luteum in less than five days after the estrus, an alternative for the reduction of the estrus interval in the beginning of the cycle would be the use of estrogen. It was used 16 Holstein-zebu crossbred cows cycling regularly, without any clinical or reproductive alterations. Cows were randomly included in their respective treatments. Eight cows received $2.5 \mathrm{~mL}$ of estradiol cipionate on the first day of the estrous cycle and eight cows did not receive anything (control). The expression of estrus was monitored visually. The ultrasound scans were conducted daily in the morning, starting on the estrus day. The blood collections for the measurements of the levels of progesterone began on the estrus day. All the animals that received estrogen on the first day after natural estrus showed the estrus characteristical signals (psychics, turgid uterus and abundant mucus) one day after application. This reduction of estrus interval to only two days can promote the elimination of uterus bacteria (if present), consequently enhancing the reproductive efficiency. Except for the the day of the first follicular wave emergence, all the other studied variables (wave number and length, characteristics of dominant and subordinate follicles and the parameters related to the corpus luteum) were not affected by the application of estrogen on the day after the estrus. The application of estradiol cipionate in crossbred cows one day after the natural estrus promote the emergence of estrus sign two days after the natural estrus, without affecting the characteristics of the next estrous cycle.
\end{abstract}

Key Words: cows, hormone, reproduction 


\section{Introdução}

O sucesso da bovinocultura leiteira está relacionado, em grande parte, à eficiência reprodutiva, pelo seu efeito direto na produção de leite e bezerros (Ferreira \& Sá, 1987). Dessa forma, o atraso na concepção de uma fêmea bovina aumenta o custo de produção e diminui o desempenho zootécnico (Ferreira, 1980). Neste contexto, as infecções uterinas representam um dos principais fatores de infertilidade e/ou esterilidade em vacas leiteiras, contribuindo para o alongamento do intervalo de partos (Ferreira \& Sá, 1987).

Vários estudos têm comprovado que a prostaglandina F2a (PGF2 $\alpha$ ) pode reduzir o intervalo de estros (em vacas com atividade ovariana luteal cíclica), auxiliando na recuperação de processos infecciosos do útero (clínicos ou subclínicos), melhorando a eficiência reprodutiva de rebanhos leiteiros (Ferreira, 1980; Heuwieser et al., 2000; Dhaliwal et al., 2001; Ferreira, 2003). Essas informações indicam que, quanto mais curto o intervalo estral, menos tempo o animal permanecerá em fase progesterônica, o que é desejável em animais com infecção uterina. No entanto, a PGF2 $\alpha$ não regride o corpo lúteo com menos de cinco dias após o estro (Vasconcelos, 2000; Moore \& Thatcher, 2006).

O uso da PGF2 $\alpha$ exógena em fase precoce do ciclo estral reduz o intervalo de estros de 21 para 7 a 8 dias. Entretanto, não foram encontrados na literatura pesquisada métodos alternativos que promovam maior redução desse intervalo. Essa condição seria benéfica no contexto da eficiência reprodutiva, uma vez que o endométrio, sob ação da progesterona, tem seus mecanismos de defesa reduzidos. Ao contrário, concentrações plasmáticas e uterinas elevadas de estrógenos aumentam a ação do sistema imune (Paisley, 1986; Dhaliwal et al., 2001).

Como a PGF2 $\alpha$ não regride o corpo lúteo com menos de cinco dias após o estro (Vasconcelos, 2000; Moore \& Thatcher, 2006), uma das alternativas para a redução do intervalo estral no início do ciclo seria o uso do estrógeno. Em determinadas fases do ciclo estral de bovinos, o estrógeno leva a regressão do corpo lúteo, supressão da secreção de hormônio luteinizante (LH) e estímulo à produção de prostaglandinas (Roberts, 1986; Pratt et al., 1991; Burke et al., 1996).

O objetivo neste trabalho foi verificar o efeito do estrógeno exógeno, em fase precoce do ciclo estral, de vacas leiteiras, na indução do estro e na dinâmica ovariana.

\section{Material e Métodos}

O experimento foi conduzido no Campo Experimental de Santa Mônica (CESM), pertencente à Embrapa Gado de
Leite e localizado no distrito de Juparanã, Município de Valença, Rio de Janeiro, no período de 1 de março a 15 de maio de 2008.

Foram utilizadas 16 vacas adultas, multíparas, mestiças Holandês \& Zebu, com graus de sangue entre 1/2 e 7/8 com predominância de Holandês, não-lactantes e não gestantes, todos os animais estavam ao final da terceira lactação. Os animais foram distribuídos em dois grupos em função do hormônio utilizado (cipionato de estradiol). Os animais do grupo 1 apresentaram peso médio de $488 \pm 35 \mathrm{~kg}$, com escore da condição corporal (ECC) médio de $3,5 \pm 0,25$. As vacas do grupo 2 apresentaram peso médio de $471 \pm 40 \mathrm{Kg}$, com ECC médio de $3,4 \pm 0,3$, na escala de 1 (muito magra) a 5 (gorda), descrita por Ferreira \& Torres (1993).

Os animais estavam ciclando regularmente, sendo selecionados por meio de exame ginecológico (palpação transretal e vaginoscopia), sendo utilizados somente os animais sem qualquer alteração clínica ou reprodutiva. As vacas foram mantidas em pastagens formadas por capimbraquiária (Brachiaria decumbens e Brachiaria brizantha), onde recebiam mistura mineral à vontade e tinham água disponível em bebedouros cimentados nos piquetes.

As vacas foram incluídas ao acaso após a detecção do estro nos seguintes tratamentos: oito vacas receberam 2,5 $\mathrm{mL}$ de cipionato de estradiol no primeiro dia do ciclo estral, via intramuscular (IM), na região da garupa, com agulha $25 \times 0,7 \mathrm{~mm}$, (considerando dia zero como dia do estro); controle: oito vacas não receberam hormônio.

A manifestação de estro foi monitorada visualmente durante uma hora por três vezes ao dia: manhã (das 6 às 7 h), meio-dia (12 às 13 h) e à tarde (18 às 19 h), com auxílio de rufião preparado por aderência peniana e equipado com buçal marcador com tinta vermelha para auxiliarnaidentificação dos animais em estro. Durante os horários de observação, quando necessário, os animais foram movimentados para agrupamento e maior contato entre si, evitando-se, assim, preferências por parte de grupos sexualmente ativos. Foram considerados em estro os animais que aceitavam monta pelo rufião ou pelas companheiras do rebanho.

Foram realizados exames ultrassonográficos por via transretal, utilizando-se aparelho portátil da marca ALOKA, modelo SSD-500, acoplado a um transdutor linear de $5 \mathrm{MHz}$. As medições tiveram início no dia do estro e foram realizadas diariamente pela manhã, por um único operador, durante um período interestral de 16 animais, totalizando 16 períodos. Foi registrado o diâmetro máximo do maior e do segundo maior folículo presente em cada ovário. Os valores obtidos foram tabulados para representação gráfica da dinâmica folicular dos animais. Ointervalo interestral (dias) foi definido como o número de dias compreendidos entre dois estros 
naturais consecutivos, caracterizado pelo comportamento estral (Rhodes et al., 1995).

A emergência da onda folicular foi estabelecida como o primeiro dia em que se encontrou um folículo com diâmetro entre 4 e $5 \mathrm{~mm}$. Cada onda foi dividida em fase de crescimento, estática e de regressão. A fase de crescimento iniciou no dia da detecção do folículo, terminando no dia em que cessou seu crescimento progressivo. A fase estática compreendeu o último dia de crescimento até o início da redução de seu diâmetro, e a de regressão, entre o último dia do diâmetro estático até atingir um diâmetro de 4 a 5 mm (Ginther et al., 1989).

O folículo dominante de cada onda foi definido como aquele que possuía o maior diâmetro, o qual excedeu o de todos os outros folículos da onda. Considerou-se apenas um folículo subordinado, por cada onda, sendo aquele que emergiu simultaneamente com o folículo dominante, porém de menor diâmetro e de menor persistência. Os diâmetros do folículo dominante e do subordinado foram medidos pela maior distância ( $\mathrm{mm}$ ) entre dois pontos da cavidade antral dos folículos, a partir de $4 \mathrm{~mm}$. Quando o folículo atingiu seu diâmetro máximo, calculou-se a duração em dias.

O comprimento da onda de crescimento folicular correspondeu ao número de dias entre sua emergência e a regressão do folículo dominante, até um diâmetro de 4 ou 5 $\mathrm{mm}$. O dia da detecção da onda folicular foi definido como o dia do primeiro registro de um folículo de diâmetro entre 4 e $5 \mathrm{~mm}$. O dia da divergência foi quando o folículo dominante e o subordinado divergiram suas curvas de crescimento.

As características morfológicas do corpo lúteo foram avaliadas diariamente ao longo do ciclo estral, medindo-se a área da seção transversal $\left(\mathrm{cm}^{2}\right)$ e das cavidades luteais (área e máximo diâmetro longitudinal e transversal), segundo os procedimentos de Pierson \& Ginther (1988). A área do tecido luteal foi calculada pela diferença entre a área da seção transversal do corpo lúteo e da cavidade luteal. O volume do tecido luteal e da cavidade do corpo lúteo foi calculada pela fórmula matemática: $V=4 / 3 \mathrm{P} . \mathrm{a} / 2 .(\mathrm{b} / 2)^{2}$, em que $\mathrm{a}=$ eixo longitudinal e $\mathrm{b}=$ eixo transversal (Grygar et al., 1997). O volume da cavidade luteal foi subtraído do volume do corpo lúteo para determinar o volume do tecido luteal em corpos lúteos cavitários.

As coletas de sangue para as dosagens de progesterona tiveram início no dia do estro (dia 0 ) e foram realizadas a cada três dias até o próximo estro. Todas as coletas foram realizadas sempre antes de qualquer procedimento. Foi considerada atividade luteal a concentração de progesterona a partir de 1,0 ng/mL (Youngquist, 1997; Wiltbank \& Niswender, 1992; Gutierrez et al., 1994; Mapletoft et al., 2000; Wiltbank et al., 2000; Gonçalves et al., 2002; González, 2002).
As amostras foram coletadas em tubos vacuolizados de $15 \mathrm{~mL}$, sem solução anticoagulante, por punção da artéria ou veia caudal e condicionados em caixa de isopor com gelo. Os tubos foram imediatamente centrifugados a $1.500 \mathrm{G}$ durante 15 minutos, para a separação do soro, que foi transferido para tubetes plásticos previamente identificados e estocados a temperatura de $-20{ }^{\circ} \mathrm{C}$ até sua análise.

As concentrações de progesterona foram determinadas usando o método de quimioluminescência com kits comercialmente disponíveis Diagnostic Products Corporation (DPC - Immunolite), dos Estados Unidos. A sensibilidade foi $0,2 \mathrm{ng} / \mathrm{mL}$ para progesterona.

As variáveis quantitativas foram submetidas aos testes de normalidade (Lilliefors) e homocedasticidade (Bartlett). Posteriormente, as médias foram comparadas pelo teste $\mathrm{F}$, adotando-se o nível de 5\% de probabilidade. Para as análises com mais de um grau de liberdade, foi realizado o teste de comparação de médias de Tukey, adotando-se o nível de 5\% de probabilidade. Quando não atendiam as premissas de normalidade e homocedasticidade, mesmo após as transformações apropriadas, os dados foram submetidos ao teste não-paramétrico de Wilcoxon (SAEG, 1999).

\section{Resultados e Discussão}

Todos os animais que receberam estrógeno no primeiro dia após o estro natural manifestaram sinais característicos de estro (psíquicos, útero túrgido e muco abundante) um dia após a sua aplicação o tratamento, sendo que seis deles apresentaram esses sinais por dois dias consecutivos. Essa redução do intervalo de estro para apenas dois dias pode ser benéfica, por favorecer a eliminação de bactérias do útero, quando presentes (Ferreira et al., 2000; Dhaliwal et al., 2001; Noakes et al., 2003). Além disso, trata-se de um medicamento de baixo custo, que permite evitar o descarte de leite pelo uso de antibióticos (Ferreira, 2003).

Excetuando o dia da emergência da primeira onda folicular, todas as outras variáveis estudadas do ciclo estral subsequente (número e comprimento de ondas, características dos folículos dominantes e subordinados, assim como os parâmetros relacionados ao corpo lúteo e produção de progesterona), não foram afetadas pela aplicação de estrógeno um dia após o estro (Tabela 1).

A dinâmica folicular das vacas mestiças (Holandês $\times$ Zebu) apresentou padrão de duas ou três ondas de crescimento folicular (Tabela 1), independentemente da aplicação do estrógeno (37,5 e 62,5\% para ciclos com duas e três ondas, respectivamente). Não houve diferença quanto ao intervalo de estro entre os animais do grupo tratado com estrógeno e controle (Tabela 1). 
Tabela 1 - Características das ondas de crescimento folicular das vacas controle e tratadas com estrógeno no início do ciclo estral

\begin{tabular}{|c|c|c|c|c|}
\hline \multirow[t]{3}{*}{ Característica } & \multicolumn{4}{|c|}{ Ondas foliculares } \\
\hline & \multicolumn{2}{|c|}{ Tratadas } & \multicolumn{2}{|c|}{ Controle } \\
\hline & 2 ondas $(\mathrm{n}=3)$ & 3 ondas $(n=5)$ & 2 ondas $(n=3)$ & 3 ondas $(n=5)$ \\
\hline Intervalo estral (dias) & \multicolumn{4}{|c|}{ Comprimento onda (dias) } \\
\hline $1 \underline{\mathrm{a}}$ onda & $14,0 \pm 2,1 \mathrm{a}, \mathrm{A}$ & $13,06 \pm 1,1 \mathrm{a}, \mathrm{A}$ & $13,87 \pm 1,8 \mathrm{a}, \mathrm{A}$ & $13,26 \pm 1,9 \mathrm{a}, \mathrm{A}$ \\
\hline 3a onda & \multicolumn{4}{|c|}{ Dia da detecção onda } \\
\hline 1ㅡa onda & $3,33 \pm 0,5 \mathrm{a}, \mathrm{A}$ & $3,43 \pm 0,4 a, A$ & $0,28 \pm 0,6 \mathrm{~b}, \mathrm{~A}$ & $0,38 \pm 0,4 \mathrm{~b}, \mathrm{~A}$ \\
\hline $2^{\underline{a}}$ onda & $9,97 \pm 1,1 \mathrm{a}, \mathrm{B}$ & $9,02 \pm 0,8 b, B$ & $9,33 \pm 1,3 a, B$ & $8,43 \pm 1,0 \mathrm{~b}, \mathrm{~B}$ \\
\hline 3a onda & - & $14,72 \pm 1,0 \mathrm{a}, \mathrm{C}$ & & $15,35 \pm 1,7 a, C$ \\
\hline
\end{tabular}

Segundo Taylor \& Rajamahendran (1991)e Guinther et al. (1996), as variações no número de ondas foliculares, estão relacionadas com a duração do ciclo estral e o tempo de vida útil do corpo lúteo. Assim sendo, quanto maior a vida útil do corpo lúteo (Tabela 2), maior será o número de ondas de crescimento folicular durante o ciclo estral.

Com relação à emergência da primeira onda de crescimento folicular, observou-se atraso nos animais do grupo tratado com estrógeno $(\mathrm{P}<0,05)$, tanto para ciclos com duas como para aqueles com três ondas foliculares, em relação ao grupo controle (Tabela 1). Resultados semelhantes aos do grupo controle foram encontrados por Borges et al. (2001), que observaram o aparecimento da primeira onda folicular nos dias 0,42 e 0,38 em novilhas mestiças com duas e três ondas foliculares, respectivamente. De acordo com O’Rourke et al. (2000) e Burke et al. (2003), o estrógeno administrado na fase de dependência do folículo ao FSH atua em receptores da hipófise anterior, bloqueando a secreção de FSH, o que provocaria atresia dos folículos dependentes desta gonadotrofina. Dessa forma, a emergência de uma nova onda é atrasada por três a cinco dias, fato confirmado neste estudo.

A emergência da segunda onda de crescimento folicular, em ciclos com duas ondas, ocorreu nos dias $9,97 \pm 1,1$ e $9,33 \pm 1,3$ para os animais dos grupos tratado e

Tabela 2 - Duração média do corpo lúteo (dias) das vacas dos grupos controle e tratadas com estrógeno no inicio do ciclo estral

\begin{tabular}{lcc}
\hline & \multicolumn{2}{c}{ Onda folicular } \\
\cline { 2 - 3 } & 2 ondas & 3 ondas \\
\hline Tratado & $15,3 \pm 1,4 \mathrm{a}$ & $17,6 \pm 1,8 \mathrm{~b}$ \\
Controle & $14,8 \pm 1,8 \mathrm{a}$ & $17,8 \pm 1,2 \mathrm{~b}$ \\
\hline
\end{tabular}

Médias com letras minúsculas diferentes sobrescritas na mesma linha são diferentes $(\mathrm{P}<0,05)$ pelo teste $\mathrm{F}$. Médias da mesma coluna não são diferentes $(\mathrm{P}>0,05)$ pelo teste F. controle, respectivamente. Esses resultados corroboram os relatados por Guinther et al. (1989), Hamilton et al. (1995), Figueiredo et al. (1997), Borges et al. (2001) e Alves et al. (2002). Entretanto, nos ciclos com três ondas foliculares, a emergência da segunda e da terceira ondas ocorreu, respectivamente, nos dias 9,02 $\pm 0,8$ e 14,72 $\pm 1,0$ (grupo tratado) e $8,43 \pm 1,0$ e $15,35 \pm 1,7$ (grupo controle), não havendo diferença entre os grupos $(\mathrm{P}>0,05)$. Esses valores estão próximos aos dias 9 e 16 dias relatados por Kastelic (1994) e 8,2 e 16 dias citados por Borges et al. (2001).

Quanto à duração das ondas foliculares, não foram encontradas diferenças, entre animais tratados e controle, em ciclos com duas ondas foliculares $(\mathrm{P}>0,05)$ (Tabela 1$)$. No presente experimento, a duração da segunda onda foi menor do que a da primeira nos animais dos dois grupos estudados $(\mathrm{P}<0,05)$. Esta condição pode ser devida à redução na função luteal (luteólise), evitando o efeito negativo da progesterona sobre o crescimento folicular, permitindo que os folículos dominantes, livres do feedback negativo, cresçam rapidamente e atinjam o tamanho ovulatório (Sávio et al., 1992; Borges et al., 2001). Nos ciclos com três ondas de crescimento folicular, não foram encontradas diferenças $(\mathrm{P}>0,05)$ na duração das ondas entre animais tratados e controle (Tabela 1$)$.

De acordo com Driancourt (2001), o estrógeno injetado precocemente após a ovulação em bovinos pode ter ação antiluteotrópica, mas isso não ocorreu neste trabalho, pois a duração da fase luteal do grupo tratado não diferiu $(\mathrm{P}>0,05)$ daquela observada no grupo controle. Além disso, o tamanho do corpo lúteo (Tabela 3) e a produção de progesterona (Tabela 4$)$ não foram diferentes $(\mathrm{P}>0,05)$ para os grupos experimentais.

O corpo lúteo foi detectado inicialmente no dia 3,9 \pm 0,9 após o estro. Entretanto, não ocorreu variação no dia da detecção do corpo lúteo entre os animais tratados ou não 
Tabela 3 - Parâmetros médios do corpo lúteo das vacas dos grupos controle e tratadas com estrógeno no inicio do ciclo estral

\begin{tabular}{lrr}
\hline Parâmetro do corpo lúteo & \multicolumn{2}{c}{ Onda folicular } \\
\cline { 2 - 3 } & \multicolumn{1}{c}{ Tratado } & \multicolumn{1}{c}{ Controle } \\
\hline Área inicial $\left(\mathrm{cm}^{2}\right)$ & $1,43 \pm 0,6 \mathrm{a}$ & $1,29 \pm 0,1 \mathrm{a}$ \\
Área final $\left(\mathrm{cm}^{2}\right)$ & $1,42 \pm 0,2 \mathrm{a}$ & $1,31 \pm 0,3 \mathrm{a}$ \\
Área máxima $\left(\mathrm{cm}^{2}\right)$ & $3,81 \pm 0,9 \mathrm{a}$ & $3,68 \pm 0,8 \mathrm{a}$ \\
Dia da área e volume máximos & $10,02 \pm 1,9 \mathrm{a}$ & $10,11 \pm 1,9 \mathrm{a}$ \\
Volume máximo $\left(\mathrm{cm}^{3}\right)$ & $4,03 \pm 1,2 \mathrm{a}$ & $4,13 \pm 0,7 \mathrm{a}$ \\
\hline Médias com letras minúsculas diferentes sobrescritas na mesma linha são diferentes
\end{tabular}
$(\mathrm{P}<0,05)$ pelo teste de $\mathrm{F}$.

Tabela 4 - Concentrações plasmáticas médias de progesterona das vacas dos grupos controle e tratadas com estrógeno no inicio do ciclo estral

\begin{tabular}{crr}
\hline Dia do ciclo estral & \multicolumn{2}{c}{ Progesterona $(\mathrm{ng} / \mathrm{mL})$} \\
\cline { 2 - 3 } & \multicolumn{1}{c}{ Tratado } & \multicolumn{1}{c}{ Controle } \\
\hline 0 & $0,32 \pm 0,6 \mathrm{a}$ & $0,21 \pm 0,6 \mathrm{a}$ \\
3 & $0,56 \pm 0,6 \mathrm{a}$ & $0,67 \pm 0,6 \mathrm{a}$ \\
6 & $3,33 \pm 1,1 \mathrm{a}$ & $4,01 \pm 1,3 \mathrm{a}$ \\
9 & $8,17 \pm 0,9 \mathrm{a}$ & $8,95 \pm 1,5 \mathrm{a}$ \\
12 & $13,85 \pm 2,1 \mathrm{a}$ & $14,23 \pm 1,6 \mathrm{a}$ \\
15 & $13,67 \pm 1,9 \mathrm{a}$ & $13,86 \pm 1,3 \mathrm{a}$ \\
18 & $5,23 \pm 3,6 \mathrm{a}$ & $4,76 \pm 2,8 \mathrm{a}$ \\
21 & $0,36 \pm 2,8 \mathrm{a}$ & $0,29 \pm 2,6 \mathrm{a}$ \\
\hline
\end{tabular}

Médias com letras minúsculas diferentes sobrescritas na mesma linha são diferentes $(\mathrm{P}<0,05)$ pelo teste $\mathrm{F}$.

com estrógeno, independentemente do número de ondas foliculares. Os resultados do presente experimento corroboram com os obtidos por Tom et al. (1998) e Coutinho et al. (2007), que identificaram o corpo lúteo pela primeira vez no quarto dia do ciclo.
Não houve diferença entre as vacas do grupo tratado e controle, assim como entre duas e três ondas (Tabela 3). A área e o volume do corpo lúteo não diferiram entre vacas apresentando duas e três ondas foliculares, mas diferiram entre os dias do ciclo estral $(\mathrm{P}<0,05)$. No acompanhamento ultrassonográfico, o tecido luteal foi detectado a partir de uma área de $1,29 \mathrm{~cm}^{2}$, valor semelhante à área do último dia em que o corpo lúteo pôde ser verificado por ultra-sonografia (Tabela 3).

Os maiores valores de progesterona foram obtidos no $12^{\underline{0}}$ dia do ciclo estral, para os dois grupos (Tabela 4). Os valores encontrados estão de acordo com a literatura, onde são citadas concentrações plasmáticas de progesterona durante o diestro de ciclos estrais normais, entre 1 e $20 \mathrm{ng} / \mathrm{mL}$ em animais das raça zebuínas e taurinas (Jiménez et al., 1988; Badinga et al., 1994; Borges et al., 2001; Alves et al., 2002; Borges et al., 2003; Coutinho et al., 2007). Essa amplitude pode ser ocasionada por variações inerentes aos animais, assim como pela metodologia de análise, fazendo com que a interpretação dos resultados de progesterona devam ser criteriosamente avaliados (Borges et al., 2001). As concentrações médias de progesterona durante o ciclo estral não diferiram entre os animais tratados com estrógeno e controle $(\mathrm{P}>0,05)$.

Após o surgimento das ondas foliculares, o folículo dominante cresceu no período de 4,32 a 8,58 dias (Tabela 5), e o diâmetro alcançado variou de 10,98 a 13,78 mm, resultados semelhantes aos encontrados por Borges et al. (2001) em novilhas mestiças Holandês $\times$ Zebu (10,0 a 13,0 mm) e inferiores aos encontrados por Guinther et al. (1989)

Tabela 5 - Comparação das características dos folículos dominantes das vacas controle e tratadas com estrógeno no inicio do ciclo estral

\begin{tabular}{|c|c|c|c|c|}
\hline \multirow{3}{*}{$\begin{array}{l}\text { Característica } \\
\text { Folículo dominante }\end{array}$} & \multicolumn{4}{|c|}{ Onda folicular } \\
\hline & \multicolumn{2}{|c|}{ Tratado } & \multicolumn{2}{|c|}{ Controle } \\
\hline & 2 ondas $(\mathrm{n}=3)$ & 3 ondas $(n=5)$ & 2 ondas $(\mathrm{n}=3)$ & 3 ondas $(n=5)$ \\
\hline \multicolumn{5}{|c|}{ Diâmetro máximo (mm) } \\
\hline $1^{0}$ folículo & $12,77 \pm 2,0 \mathrm{a}, \mathrm{A}$ & $12,52 \pm 1,8 a, A$ & $13,07 \pm 2,8 a, A$ & $12,94 \pm 2,0 \mathrm{a}, \mathrm{A}$ \\
\hline 2o folículo & $13,78 \pm 1,7 a, A$ & $11,01 \pm 1,3 \mathrm{~b}, \mathrm{~B}$ & $13,17 \pm 1,1 \mathrm{a}, \mathrm{A}$ & $10,98 \pm 1,2 \mathrm{~b}, \mathrm{~B}$ \\
\hline 3o folículo & - & $12,86 \pm 1,3 a, A$ & - & $12,16 \pm 1,1 \mathrm{a}, \mathrm{A}$ \\
\hline \multicolumn{5}{|c|}{ Dia do diâmetro máximo } \\
\hline $1^{10}$ folículo & $8,12 \pm 1,8 a, A$ & $7,75 \pm 1,0 \mathrm{a}, \mathrm{A}$ & $6,82 \pm 1,3 a, A$ & $6,62 \pm 1,5 \mathrm{a}, \mathrm{A}$ \\
\hline $2^{2}$ folículo & $18,0 \pm 2,0 \mathrm{a}, \mathrm{B}$ & $12,46 \pm 2,7 \mathrm{~b}, \mathrm{~B}$ & $18,12 \pm 1,8 a, B$ & $13,06 \pm 2,1 \mathrm{~b}, \mathrm{~B}$ \\
\hline 3 o folículo & - & $20,09 \pm 1,3 a, C$ & - & $20,28 \pm 1,0 \mathrm{a}, \mathrm{C}$ \\
\hline \multicolumn{5}{|c|}{ Duração do crescimento (dias) } \\
\hline 1ํㅡ folículo & $4,82 \pm 1,8 \mathrm{a}, \mathrm{A}$ & $4,32 \pm 1,0 \mathrm{a}, \mathrm{A}$ & $6,52 \pm 1,1 \mathrm{a}, \mathrm{A}$ & $6,51 \pm 1,5 \mathrm{a}, \mathrm{A}$ \\
\hline 2o folículo & $8,58 \pm 1,3 a, B$ & $5,12 \pm 2,3 \mathrm{~b}, \mathrm{~B}$ & $8,11 \pm 1,9 a, B$ & $5,23 \pm 1,9 b, B$ \\
\hline 3 o folículo & - & $5,23 \pm 1,1 \mathrm{a}, \mathrm{B}$ & - & $5,31 \pm 1,2 \mathrm{a}, \mathrm{B}$ \\
\hline \multicolumn{5}{|l|}{ Início da atresia (dia) } \\
\hline 1 o folículo & $9,02 \pm 2,6 a$ & $9,18 \pm 2,2 \mathrm{a}, \mathrm{A}$ & $9,04 \pm 1,1 \mathrm{a}$ & $8,15 \pm 2,0 \mathrm{a}, \mathrm{A}$ \\
\hline $2^{2}$ folículo & - & $15,24 \pm 1,9 a, B$ & - & $16,01 \pm 2,3 \mathrm{a}, \mathrm{B}$ \\
\hline 3 o folículo & - & - & - & - \\
\hline
\end{tabular}

Médias com letras minúsculas diferentes sobrescritas na mesma linha são diferentes $(\mathrm{P}<0,05)$ pelo teste Tukey ou $\mathrm{F}$.

Médias com letras maiúsculas diferentes sobrescritas na mesma coluna são diferentes $(\mathrm{P}<0,05)$ pelo teste Tukey ou $\mathrm{F}$. 
para animais da subespécie Bos taurus taurus (13 a 18 mm). Os menores valores em relação aos taurinos podem estar relacionados à menor taxa de crescimento dos folículos dominantes de vacas de raças zebuínas (1,33 mm/dia), segundo Coutinho et al. (2007) em comparação à de vacas das raças europeias (2,05 mm/dia; Murphy et al., 1990).

De acordo com Borges et al. (2001), uma possível explicação para a diferença nos diâmetros foliculares seria a concentração plasmática de progesterona. A primeira onda folicular coincide com baixa concentração deste hormônio (corpo lúteo em formação). Dessa forma, ocorre um fraco feedback negativo ao hipotálamo-hipófise, o qual é insuficiente para impedir a liberação de LH. A segunda onda inicia na presença de um corpo lúteo totalmente formado, o qual secreta elevadas concentrações de progesterona, inibindo a liberação de LH (Savio et al., 1992). A terceira onda coincide com a luteólise e consequente redução da secreção de progesterona pelo corpo lúteo. Diante disto, ocorre um incremento na secreção de hormônio luteinizante, acompanhada por um rápido crescimento folicular e da ovulação (Savio et al., 1992).

Os folículos subordinados atingem diâmetro menor que os folículos dominantes, independentemente do número de ondas foliculares (Tabela 6).

O diâmetro máximo dos folículos subordinados variou de 6,83 a 7,91 mm, valores próximos àqueles citados para animais das raças Nelore (Figueiredo et al., 1997), Holandesa (Fortune, 1994) e para novilhas mestiças
(Hol × Zebu) (Borges et al., 2001). Segundo Borges et al. (2001), o menor crescimento dos folículos subordinados ocorre devido ao efeito inibitório do folículo dominante, o qual impede um aporte adequado de gonadotrofinas e secreta um fator que tem efeito inibitório em seu desenvolvimento (Webb \& Armstrong, 1998; Bakers \& Spears, 1999). Esta inibição ocorre de forma passiva (redução nas concentrações plasmáticas de FSH) e ativa (redução da sensibilidade ao FSH). As células da granulosa do folículo dominante produzem estradiol e inibina, os quais reduzem a liberação de FSH a um limiar insuficiente para manter o desenvolvimento dos folículos subordinados. Todavia, nos folículos dominantes, determinados fatores de crescimento amplificam a ação do FSH, possibilitando-os a continuar o crescimento, mesmo sob baixos níveis hormonais (Fortune et al., 1994; Driancourt, 2001).

Partindo da premissa de que a redução do intervalo de estros é indicada como eficiente tratamento de infecções uterinas em vacas com atividade luteal cíclica, segundo Ferreira (1980) e Dhaliwal et al. (2001), e que a aplicação de estrógeno um dia após o estro natural de bovino provocou o comportamento de estro (psíquicos, muco abundante e útero túrgido) dois dias após o estro natural, sem afetar as características do ciclo estral subseqüente, deduz-se que esse procedimento pode ser recomendado para esse tipo de patologia em substituição ou associado ao que é amplamente preconizado atualmente, ou seja, a aplicação de PGF2 $\alpha 6$ a 7 dias após o estro.

Tabela 6 - Comparação das características dos folículos subordinados das vacas controle e tratadas com estrógeno no início do ciclo estral

\begin{tabular}{|c|c|c|c|c|}
\hline \multirow{3}{*}{$\begin{array}{l}\text { Característica dos } \\
\text { folículos subordinados }\end{array}$} & \multicolumn{4}{|c|}{ Onda folicular } \\
\hline & \multicolumn{2}{|c|}{ Tratado } & \multicolumn{2}{|c|}{ Controle } \\
\hline & 2 ondas $(n=3)$ & 3 ondas $(n=5)$ & 2 ondas $(\mathrm{n}=3)$ & 3 ondas $(n=5)$ \\
\hline \multicolumn{5}{|l|}{ Diâmetro máximo (mm) } \\
\hline 1ํ folículo & $7,71 \pm 1,0 \mathrm{a}, \mathrm{A}$ & $7,06 \pm 1,2 \mathrm{a}, \mathrm{A}$ & $7,32 \pm 1,4 a, A$ & $7,91 \pm 1,0 \mathrm{a}, \mathrm{A}$ \\
\hline 2o folículo & $7,03 \pm 2,0 a, A$ & $6,97 \pm 1,0 \mathrm{a}, \mathrm{A}$ & $6,83 \pm 2,4 \mathrm{a}, \mathrm{A}$ & $6,89 \pm 1,0 \mathrm{a}, \mathrm{A}$ \\
\hline 3 o folículo & - & $7,08 \pm 1,1 \mathrm{a}, \mathrm{A}$ & - & $6,98 \pm 1,2 \mathrm{a}, \mathrm{A}$ \\
\hline \multicolumn{5}{|l|}{ Dia do diâmetro máximo } \\
\hline $1^{\text {o }}$ folículo & $6,02 \pm 1,1 \mathrm{a}, \mathrm{A}$ & $5,96 \pm 1,9 a, A$ & $3,08 \pm 1,0 \mathrm{~b}, \mathrm{~A}$ & $2,88 \pm 1,8 \mathrm{~b}, \mathrm{~A}$ \\
\hline $2^{2}$ folículo & $13,33 \pm 1,9 a, B$ & $10,91 \pm 1,6 b, B$ & $12,93 \pm 1,5 \mathrm{a}, \mathrm{B}$ & $11,01 \pm 1,3 \mathrm{~b}, \mathrm{~B}$ \\
\hline $3^{0}$ folículo & - & $17,91 \pm 1,5 a, C$ & - & $18,07 \pm 1,3 a, C$ \\
\hline \multicolumn{5}{|c|}{ Duração do crescimento (dias) } \\
\hline 1ํ folículo & $3,67 \pm 1,1 \mathrm{a}, \mathrm{A}$ & $3,51 \pm 1,8 \mathrm{a}, \mathrm{A}$ & $3,67 \pm 1,1 \mathrm{a}, \mathrm{A}$ & $2,32 \pm 1,5 \mathrm{a}, \mathrm{A}$ \\
\hline 2o folículo & $3,28 \pm 1,1 \mathrm{a}, \mathrm{A}$ & $3,10 \pm 1,2 \mathrm{a}, \mathrm{A}$ & $3,28 \pm 1,1 \mathrm{a}, \mathrm{A}$ & $2,92 \pm 1,8 \mathrm{a}, \mathrm{A}$ \\
\hline 3 o folículo & - & $2,89 \pm 1,6 a, A$ & - & $2,87 \pm 1,0 \mathrm{a}, \mathrm{A}$ \\
\hline \multicolumn{5}{|l|}{ Início da atresia (dia) } \\
\hline 1ํㅡ folículo & $7,02 \pm 1,3 a, A$ & $6,24 \pm 1,6 a, A$ & $4,02 \pm 1,5 \mathrm{~b}, \mathrm{~A}$ & $4,06 \pm 1,2 \mathrm{~b}, \mathrm{~A}$ \\
\hline $2^{0}$ folículo & $13,05 \pm 2,0 \mathrm{a}, \mathrm{B}$ & $12,34 \pm 1,4 \mathrm{~b}, \mathrm{~B}$ & $13,05 \pm 1,7 \mathrm{a}, \mathrm{B}$ & $12,06 \pm 2,0 \mathrm{~b}, \mathrm{~B}$ \\
\hline 3 o folículo & - & $18,04 \pm 1,4 a, C$ & - & $18,93 \pm 1,8 a, C$ \\
\hline
\end{tabular}

Médias com letras minúsculas diferentes sobrescritas na mesma linha são diferentes $(\mathrm{P}<0,05)$ pelo teste Tukey ou $\mathrm{F}$.

Médias com letras maiúsculas diferentes sobrescritas na mesma coluna são diferentes $(P<0,05)$ pelo teste Tukey ou $F$. 


\section{Conclusões}

A aplicação de cipionato de estradiol em vacas mestiças (Holandês $\times$ Zebu) um dia após o estro natural promove o aparecimento de sinais de estro (psíquicos, útero túrgido e muco abundante) dois dias após o estro natural, sem afetar as características do ciclo estral subsequente (duração do ciclo, número de ondas, tamanho dos folículos dominantes e subordinados, tamanho do corpo lúteo e produção de progesterona).

\section{Referências}

ALVES, N.G.; COSTA, E.P.; GUIMARÃES, J.D. et al. Atividade ovariana em fêmeas bovinas da raça Holandesa e Mestiças Holandês $\times$ Zebu, durante dois ciclos estrais normais consecutivos. Revista Brasileira de Zootecnia, v.31, p.627-634, 2002.

BADINGA, L.; THATCHER, W.W.; WILCOX, C.J. et al. Effect of season on follicular dynamics and plasma concentrations of estradiol-17b, progesterone and luteinizing hormone in lactating Holstein cows. Theriogenology, v.42, p.1263-1274, 1994.

BAKER, S.J.; SPEARS, N. The role of intra-ovarian interactions in the regulation of follicle dominance. Human Reproduction Update, v.5, p.153-165, 1999.

BORGES, A.M.; TORRES, C.A.A.; RUAS, J.R.M. et al. Dinâmica follicular ovariana em novilhas mestiças Holandês-Zebu, Arquivo Brasileiro de Medicina Veterinária e Zootecnia, v.53, n.5, p.595-604, 2001.

BORGES, Á.M.; TORRES, C.A.A.; RUAS, J.R.M. et al. Características da dinâmica folicular e regressão luteal de vacas das racas Gir e Nelore após tratamento com cloprostenol sódico. Revista Brasileira de Zootecnia, v.32, n.1, p.85-92, 2003.

BURKE, C.R.; MACMILLAN, K.L.; BOLAND, M.P. Estradiol potentiates a prolonged progesterone-induced suppression of LH release in ovariectomised cows. Animal Reproduction Science, v.45, p.13-28, 1996.

BURKE, C.R.; MUSSARD, C.L.; GASSER, D.E. et al. Estradiol benzoate delays new follicular wave emergence in a dosedependent manner after ablation of the dominant ovarian follicle in cattle. Theriogenology, v.60, p.647, 2003.

COUTINHO, G.T.R.M.; VIANA, J.H.M.; SÁ, W.F. et al. Avaliação ultra-sonográfica da dinâmica folicular e lútea em vacas da raça Guzerá. Arquivo Brasileiro de Medicina Veterinária e Zootecnia, v.59, n.5, p.1089-1096, 2007.

DHALIWAL, G.S.; MURRAY, R.D.; WOLDDEHWET, Z. Some aspects of immunology of the bovine uterus related to treatments for endometritis. Animal Reproduction Science, v.67, n.3-4, p.135-152, 2001.

DRIANCOURT, J.C. Regulation of ovarian follicular dynamics in farm animals. Implications for manipulation of reproduction. Theriogenology, v.55, p.1211-1239, 2001.

FERREIRA, A.M. Efeito do cloprostenol no tratamento da metrite bovina. 1980. 134f. Dissertação (Mestrado em Medicina Veterinária) - Universidade Federal de Minas Gerais, Belo Horizonte.

FERREIRA, A.M.; SÁ, W.F. Estudo das infecções uterinas em vacas leiteiras. Pesquisa Agropecuária Brasileira, v.22, n.3, p.339-344, 1987.

FERREIRA, A.M.; TORRES, C.A.A. Perda de peso corporal e cessação da atividade ovariana luteínica cíclica em vacas mestiças leiteiras. Pesquisa Agropecuária Brasileira, v.28, p.411-418, 1993.
FERREIRA, A.M.; SÁ, W.F.; COSTA, E.P. et al. Corpo lúteo persistente associado a infecções uterinas em rebanhos leiteiros da Zona da Mata-MG. Revista Brasileira de Ciência Veterinária, v.7, n.1, p.25-28, 2000.

FERREIRA, A. M. Informações pessoais. Embrapa Gado de Leite, 2003.

FIGUEIREDO, R.A; BARROS, C.M.; PINHEIRO, O.L. et al. Ovarian follicular dynamics in Nelore breed (Bos indicus) cattle. Theriogenology, v.47, p.1489-1505, 1997.

FORTUNE, J.E. Ovarian follicular growth and development in mammals. Biology of Reproduction, v.50, p.225-232, 1994.

GONÇALVES, P.B.D.; FIGUEIREDO, J.R.; FREITAS, F.J.F. Biotécnicas aplicadas à reprodução animal: controle do estro e da ovulação em bovinos e ovinos. São Paulo: Varela, 2002. 640p.

GONZÁLEZ, F.H.D. [2002]. Introdução a endocrinologia reprodutiva veterinária. Disponível em: <www.ufrgs.br/ufrgs $>$. Acesso em: 25/6/2008.

GINTHER, O.J.; KNOPF, L.; KASTELIC, J.P. Temporal associations among ovarian events in cattle during oestrus cycles with two and three follicular waves. Journal of Reproduction and Fertility, v.87, p.223-230, 1989.

GINTHER, O.J.; WILTBANK, M.C.; FRICKE,P.M. et al. Selection of dominant follicle in cattle. Biology of Reprodution, v.55, p.1187-1194, 1996.

GRYGAR, I.; KUDLÁC, E.; DOLEZEF, R. et al. Volume of luteal tissue and concentration of serum progesterone in cows bearing homogeneous corpus luteum or corpus luteum cavity. Animal Reproduction Science, v.49, p.77-82, 1997.

GUTTIERREZ, A.C.; ZARCO, L.; GALLINA, C.S. et al. Predictive value of palpation per rectum for detection of the Corpo Lúteo in zebu cattle as evaluated by progesterone concentrations and ultrassonography. Theriogenology, v.46, p.471-479, 1994.

HAMILTON, S.A; GARVERICK, H.A; KEISLER, D.H. et al. Characterization of ovarian follicular cystis and associated endocrine profiles in dairy cows. Biology of Reprodution, v.53, p.890-898, 1995.

HEUWIESER, W.; TENHAGEN, B.A.; TISCHER, M. et al. Effect of thrue programmes for the treatment of endometrits on the reproductive performance of dairy herds. Veterinary Record, v.146, p.338-341, 2000.

JIMÉNEZ, F.; GALINA, C.S.; DUCHATEAU, A. et al. Levels of $\mathrm{LH}$, porgesterone and estradiol-17b during natural and $\mathrm{PGF}_{2} \alpha$ induced estrus in Indubrazil and Brown Swiss cows in the tropics. Animal Reproduction Science, v.16, p.199-206, 1988.

KASTELIC, J.P. Understanding ovarian follicular development in cattle. Veterinary Medicine, p.64-71, 1994.

MAPLETOFT, R.J.; BO, G.A.; ADAMS, G.P. Avanços na manipulação de doadoras e receptoras nos programas de Transferência de Embriões em bovinos. Arquivo da Facudade Veterinária, v.28, n.1, p.24-51, 2000.

MOORE, K.; THATCHER, W.W. Major advances associated with reproduction in dairy cattle. Journal Dairy Science, v.89, p.1254-1266, 2006.

MURPHY, M.G.; BOLAND, M.P.; ROCHE, J.F. Pattern of follicular growth and resumption of ovarian activity in post-partum beef suckler cows. Journal of Reproduction e Fertility, v.90, p.523-533, 1990.

NOAKES, D.E.; PARKINSON, T.J.; ENGLAND, G.C.W. Infertility in the cow structural and function anormalities, management eficiences and non-specific infections. In: NOAKES, D.E.; PARKINSON, T.J.; ENGLAND, G.C.W. (Eds.) Veterinary reproduction and obstetrics. 8.ed. London: Saunders, 2003, p.383-472.

O’ROURKE, M.; DISKIN, M.; GREENAN, J.M. et al. The effect of dose and method of oestradiol administration on plasma concentration of oestradiol and FSH in long-term ovariectomised heifers. Animal Reproduction Science, v.59, p.1-12, 2000. 
PAISLEY, L.G. Mechanism and therapy for retained fetal membranes and uterine infections in the cow. Theriogenology, v.25, p.353-381, 1986.

PIERSON, R.A.; GINTHER, O.J. Ultrasonic imaging of the ovaries and uterus in cattle. Theriogenology, v.29, p.21-37, 1988.

PRATT, S.L.; SPITZER, J.C.; BURNS, G.L. et al. Luteal function, estrus response, and pregnancy rate after treatment with norgestomet and various dosages of estradiol valerate in suckled cows. Journal Animal Science, v.69, 2721-2726, 1991.

RHODES, F.M.; FITZPATRICK, L.A.; ENTWISTLE, K.W. et al. Sequential changes in ovarian follicular dynamics in Bos indicus heifers before and after nutritional anoestrus. Journal of Reproduction and Fertility, v.104, n.1, p.41-49, 1995.

ROBERTS, J.S. Veterinary obstetrics and genital diseases. Theriogenology, p.398-427, 1986.

SAVIO, J.D.; THATCHER, W.W.; BADINGA, L. et al. Regulation of dominant follicle turnover during the oestrous cycle in cows. Journal of Reproduction and Fertility, v.97, p.197-203, 1992.

TOM, J.W.; PIERSON, R.A.; ADAMS, G.P. Quantita tive echotexture analyses of bovine corpora lutea. Theriogenology, v.49, p.1345-1352, 1998.

TAYLOR, C.; RAJAMAHENDRAN, R. Follicular dynamics, corpus luteum growth and regression in lactaging dairy cattle. Canadian Journal of Animal Science, v.71, n.1, p.61-68, 1991.
UNIVERSIDADE FEDERAL DE VIÇOSA - UFV. Sistema de análise estatística e genética - SAEG. Versão 8.0. Viçosa, MG: 2000. 142p.

VASCONCELOS, J.L.M. Hormônios para sincronização do estro em vacas. MILK POINT. Radares técnicos, reprodução. 2p. Disponível em: <http://www.milkpoint.com.br/artigos-tecnicos/ reproducao>. Acesso em: 20/4/2000.

WEBB, R; ARMSTRONG, D.G. Control of ovarian function: effect of local interactions and environmental influences on follicular turnover in cattle: a review. Livestock Production Science, v.53, p.95-112,1998.

WILTBANK, M.C.; NIESWENDER, G.D. Functional aspects of differentiation and degeneration of the steroidogenic cells of the corpus luteum in domestic ruminants. Animal Reproduction Science, v.28, p.103-110, 1992.

WILTBANK, M.C.; GALLAGHER, K.P.; CHRISTENSEN, A.K. et al. Physiological and immunocytochemical evidence for a new concept of blood for regulation in the corpus luteum. Biology Reproduction, v.42, p.139-149, 2000.

YOUNGQUIST, R.S. Current therapy in large animal. In: YOUNGQUIST, R.S. (Ed.) Theriogenology - Clinical reproductive physiology of the cow. Philadelphia: Saunders Company, 1997. p.257-267. 